5. fañler.blattau

Gefhthte ber beuthen muft 


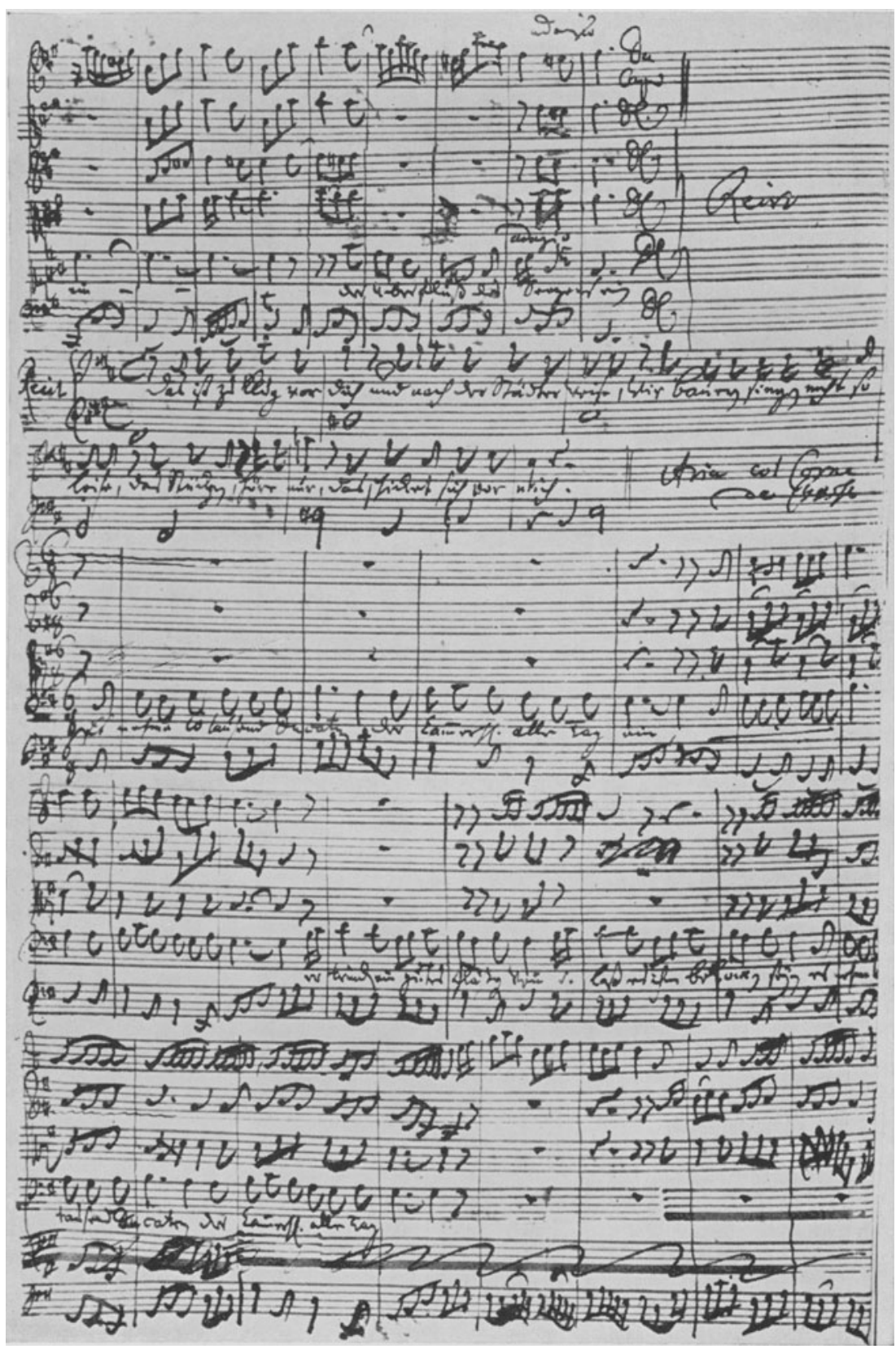

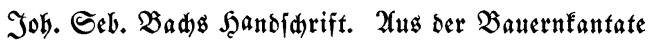

(Preū. Etaatsbibliothet, $\mathfrak{B e r l i n}$ ) 


\section{Gef fhichte}

\section{Der Deutfihen $\mathfrak{A l u f i t}$}

Bot

\section{Doref Mủllersłlattau}

mit zablrethen Potenbetptelen

1938

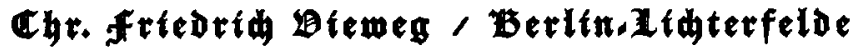


ISBN 978-3-663-00580-3 ISBN 978-3-663-02493-4 (eBook)

DOI 10.1007/978-3-663-02493-4

Softcover reprint of the hardcover 1st edition 1938

Titel zeidnung und Einband yon Frił Stübing

Alle Redte vorbehalten

Seclam-Drud Icipzig 


\section{$\mathbb{I n \mathfrak { h } a \mathfrak { l t }}$}

Seite

Borwort

Die Mufif in altgermanifder Beit . . . . . . . . 9

Die Mufif bes beutiden Mittelalters (bis zum Zob Lutbers unb Senfis)

Das Erbe ber $\mathfrak{B o r z e i t . ~ S p i e l m a n n d u n f t ~ u n d ~} \mathfrak{B}$ olfalied . . .

Die Mufif ber driftliden Kirdbe. Das geiftlide Sied in beutider Sprade . . . . . . . . . . . . . . . . 30

Die Mufit bes $\mathfrak{R i t t e r t u m s . ~}$ Bom Minnejang zum Meiftergejang 44

Die 2 Anfänge der bürgerliden Mufif und Mufifpflege . . . . 60

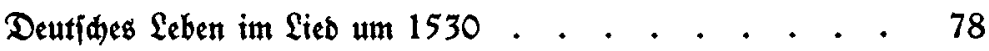

Die Mufif im Beitalter des groben Sirieges (yon Şafier

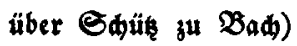

Der $\mathcal{A}$ ufbrud Der Inftrumentalmufft. Das neue Berhältnis yon Wort und $\mathfrak{Z}$ on . . . . . . . . . . . . . . 93

Die grofen Meifter: Sdakin, Sdetbt, Shür . . . . . . 105

Die beutiden Stämme und Sandjhaften im Sied . . . . . 120

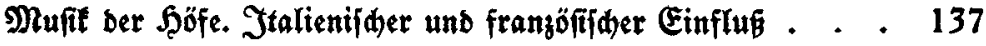

Die $\mathfrak{M u f i t}$ ber beutiden Kantoren und Drganiften . . . . 148

Bach und Şändel . . . . . . . . . . . . . . 158 
Die Mufif der Goethezeit (1750-1830)

Beite

Die 2Itergfolgen ber Komponiften . . . . . . . . . 172

Renner und Liebbaber. Die groken @eitgedanfen . . . . . 189

Saaybn - Mozart - Beetboben. Deutide Rlajfit . . . . 197

Das beutide Sied. Franz Shubert, Sart Soeme. . . . . 223

Die beutide Dper und das beutide Draforium . . . . . . 240

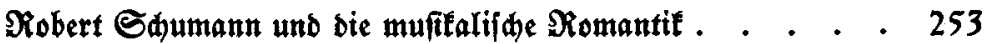

Die Formen ber bürgerliden Mufitultur . . . . . . . . 261

Verfall und Erneuerung ber beutíden Mufif (bon 1830 bis zur Gegenwart)

Franz Sijzt und ber mulitalifide Fortidgritt . . . . . . . 270

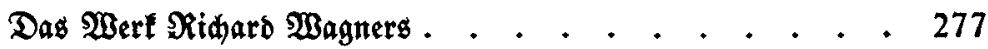

Die „âbjolute Muffit". Bruf́ner uno Brahms . . . . . . 286

Das beutfde Mufiteben und bas Erbe beutfder Mufí . . . 293

Von Gegenwart und Bukunft beutider $\mathfrak{M u f t}$. . . . . . 305

Regifiter . . . . . . . . . . . . . 311 


\section{Dormort}

Gine neue cinbänbige, Gandlide Sefdidtete ber beutiden Mufit ift gerabe in unierer Gegenwart hodnotwendig. Immer wieber wurbe in Sdulung:s. lagern, bei $\mathfrak{B o r t r a ̈ g e n , ~ i m ~ U n n t e r r i d , t ~ a u s ~ b e n ~} \Re e i h e n$ unjerer jungen Bene= ration eine folde Daritellung geforbert. Sie foll bie Sendung ber $\mathfrak{M} \mathfrak{u} ;$ i

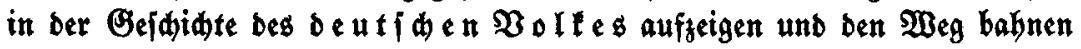

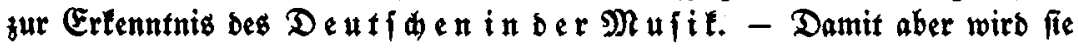
zugleid) von einer neuen Einngebung ber Mufitgejdid)te zeugen, indem fie żur flärenden Foridaung und beutenden Darftellung bie völfifde $\mathfrak{\text { Bertung }}$ Ginzufügt. Dum erft wirb der Erbftrom der Beididte lebendig in bie Gegen= wart einftrömen, bie $\mathfrak{B e r a n t w o r t u n g ~ f u ̈ r ~ f i e ~ w e d ̛ e n ~ u n b ~ b e n ~} \mathfrak{B i l l e n}$ zur $\mathbf{Z}^{\mathfrak{t}}$ s funft feftigen und befeuern. Das Sdiffal ber Mufif ift vom Sdidfal umieres Bolfes nid)t zu trennen. - So etwa war die Bieljekzung gegeben!

"İ hab'z gewagt mit Sinnen" barf id nun jagen. 2 . h. Sitehl, ber als

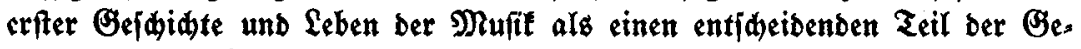
fdidte und bes Lebens unjeres beutiden $\mathfrak{B}$ olfes jah und bejhrieb, wurbe mein Borbilb. Seinem Zndenten wisme id bies $\mathfrak{W e r t .}$

Die großßen 2 nnegungen, die bon $\mathfrak{h}$. I. Mojers wagenutiger breibändiger "Geididte Der Deutiden Mufiz" auggingen, find ebenjo bedadtjam genukt. Die Sefer unjeres 2 budes werben bort, wo fie fid in Einzelfeiten ausfübrlider unterridten wollen, gern zu biejem $\mathfrak{\text { Bert }}$ greifen. Dak unjer $\mathfrak{B}$ ud als

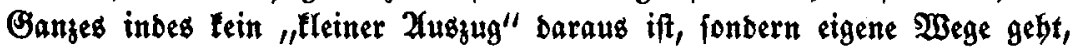
zeigt allein idon bie Glitederung bes Juhbalts.

Dic beutide Mufit ît nirgend für fid allein betrad)tet, fondern ftets als

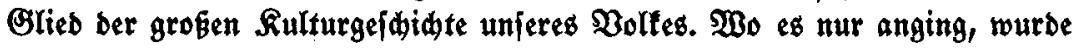
yom lebendigen Beifpiel ausgegangen. Ronnte ein foldes wegen feines $\mathfrak{u}_{\mathfrak{m}}$. fangs nid)t ganz abgebrufft werben, bann ift im Siterafurberzeidnis am Ende

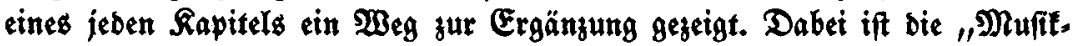
geidid)te in Beifpielen" yon $\mathfrak{A}$ rnold Shering (zitiert als Sd.) als bas einzig borbildtide Werl diejer 2 art burd Nummernangabe immer wieber herans.

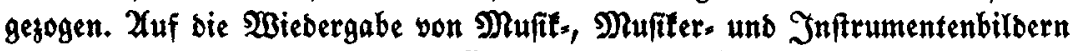
wurbe versidtet, bafür aber am Enbe eines jeben Rapitels auf entfpredende Tafeln ber "Mulitgeididite in $\mathfrak{B i l d e r n " ~ ( j i t t e r t ~ a l s ~} \mathfrak{M}$. $\mathfrak{i}$. B.) bingewiejen,

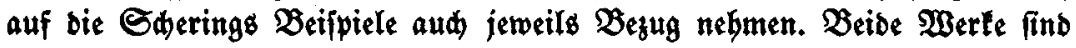


bei $\mathfrak{B r e i t f o p f}$ und Şärtel eridienen und in jeder Bibliothę vorbanden. Für

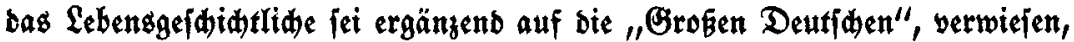

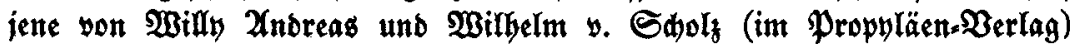
herausgegebene vierbänbige Sammlung yon Lebensbejhreibungen grober Männer, in Deren Meike bie Meifter ber beutidgen Mufif würdig bertreten find. Die Einzelbeträge find in unjernt Siteraturberzeidnis nidht einzeln aufgeführt.

Mit ber 2 Biebergabe der Shandfarift einiger grober Meifter foll auf $\mathfrak{B}_{\mathfrak{l}}=$ Deutung und Eigenart ber mufilalifden $\mathfrak{u}$ riddriften aufmerfiam gemadt werben. Die Mufiter-Sandidjriften von $\mathfrak{B a d}$ bis Sdumann, bie Georg Sdünemann im 2tlantis.Berlag herausgab und befprad, bieten weiteres, uneriab̈pflides Material.

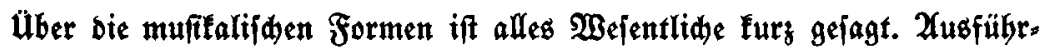
lide $\mathfrak{A}_{n}$ alyjen und eine mit vielen Beifpielen belegte Einführung in bie

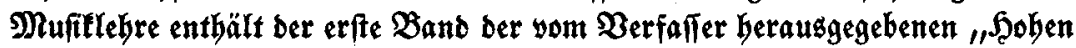

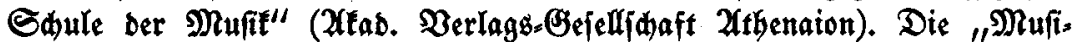

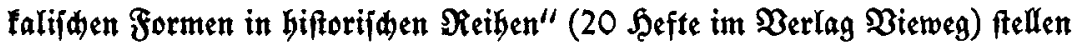
bie Beididte ber widtigften Formen mit einer Fülle bon Beijpielen bar.

Im ganzen mendet fidh unfere Bejdidjte ber beutiden Mufif ohme fad. liđe Bejđränfung, aber aud obne billige Popularität an ben Stubierenden, Den Mufíter und Mufiflehrer, Den Mufftefreund, ben in ber Sdulungbarbeif Stehenben und barüber hinaus an alle biejenigen, denen bie Pflege ber

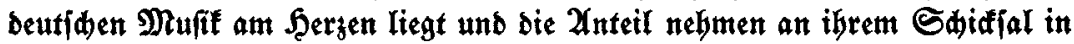
Sejobidte, Begenwart uno 3ufunft. Gewählt, bejdrieben und gewertet ift

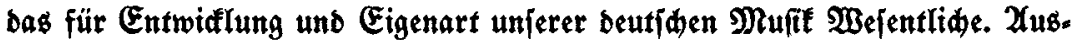
fübrlid ift gerade bie $\mathfrak{B o r}$ und Frübgefdidte behandelt; wer fid hier über

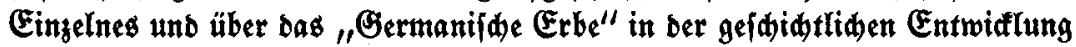
ber beutiden Mufí unterridten will, ber greife zu bes Berfafierz Sonder: baritellung unter biefem Titel im gleiden Berlage. Im weiteren Fortgang aber ift immer wieber bas Gleidgewid)t erftrebt zwifden ber Beidreibung bes zufunftsträdtigen Sdyaffens unierer großen Meifter, der Darftellung Des ganzen Mufifftandes Der betreffenden Beit und ber Errenntnis ber unauflöbliden $\mathfrak{B e r b u n d e n b e t}$ ber Mufit mit ber Gefamtgejdidbte bes Deutiden $\mathfrak{\text { Bolfes. }}$

In biejer feiner Eigenart foll bas $\mathfrak{B}_{\mathfrak{h}}$ z) zu ernfter $\mathfrak{B}$ efinnung, zu cin. bringender Selbftbejäajtigung und frudtbarer (Semeinjdaftsarbeit anregen. Möge es freubige und bereite Sefer finden!

$\mathfrak{F r e i b u r g}$ i. $\mathfrak{B r}$. im Mai 1938

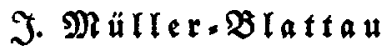

